\title{
My patient is short of breath: is there pleural fluid, and will PoCUS help drain it safely?
}

\author{
Osama Loubani' ${ }^{1}$, Justin Bowra ${ }^{2}$, Andrew Smith ${ }^{3}$, Jo Ann Talbot ${ }^{4}$, Bob Jarman ${ }^{5}$ \\ and Paul Atkinson ${ }^{4}$ \\ ${ }^{1}$ Emergency Medicine, Dalhousie University, Halifax, Nova Scotia, B3H 4R2, Canada; ${ }^{2}$ Emergency Medicine, Sydney Adventist Hospital \\ and Royal North Shore Hospital, Wahroonga, New South Wales, 2076/2065, Australia; ${ }^{3}$ Emergency Medicine, Memorial University, \\ St John's, Newfoundland, A1B 3U6, Canada; ${ }^{4}$ Emergency Medicine, Dalhousie University, Saint John Regional Hospital, Saint John, \\ New Brunswick, E2L 3L6, Canada; ${ }^{5}$ Emergency Medicine, Queen Elizabeth Hospital, Gateshead, Northeast England, NE9 6SX, UK \\ Corresponding author: Paul Atkinson. Email: Paul.Atkinson@HorizonNB.ca
}

\begin{abstract}
Pathological pleural fluid is common in patients presenting to the emergency department, occurring in as many as $17 \%$ of patients presenting with shortness of breath, and as many as $20 \%$ of patients with blunt thoracic trauma. A typical chest X-ray may fail to identify as much as $175 \mathrm{~mL}$ of pleural fluid in the erect position, and as much as $500 \mathrm{~mL}$ in the supine position. Point-of-care ultrasound (PoCUS) on the other hand can detect as little as $20 \mathrm{~mL}$ of pleural fluid, and has consistently been shown to have sensitivities and specificities for the detection of pleural fluid close to $100 \%$ in both the trauma and critically ill populations. In addition, ultrasound identifies pleural fluid more rapidly than chest X-ray. PoCUS can be used to guide thoracentesis, resulting in improved success rates with decreased complications. Here we describe the evidence supporting the use of PoCUS in the management of pleural fluid collections.
\end{abstract}

Keywords: Point of Care Ultrasound, PoCUS, Pleural Fluid, Needle guidance, Thoracentesis, Emergency Medicine

Ultrasound 2013; 0: 1-5. DOI: 10.1177/1742271X13486266

\section{Clinical questions}

In patients presenting with shortness of breath, can I use point-of-care ultrasound (PoCUS) to identify pleural fluid and does it provide any advantage over chest $\mathrm{X}$-ray (CXR)? Once detected, should I use PoCUS to guide thoracentesis?

\section{Introduction}

In healthy individuals, there is less than $10 \mathrm{~mL}$ of fluid in the pleural cavity, functioning as a lubricant between the visceral and parietal pleura. ${ }^{1}$ Abnormal accumulation of fluid may result from a variety of disease processes, including malignancy, heart failure, pneumonia, empyema and traumatic bleeding. Excessive fluid in the pleural space can lead to reduced lung volume, abnormalities in oxygenation or ventilation, and act as a nidus for infection.

Pleural effusions are common in patients presenting with respiratory symptoms. In one study of 880 patients presenting to emergency departments (ED) in North America and Europe, with a chief complaint of shortness of breath, 17\% were found to have pleural effusions. ${ }^{2}$ As many as $62 \%$ of patients requiring admission to medical intensive care unit (ICU) have pleural effusions, ${ }^{3}$ while $10-20 \%{ }^{4-10}$ of thoracic trauma patients have a haemothorax.

The presence of pleural fluid has traditionally been diagnosed through physical examination and CXR. Classical physical examination findings of pleural fluid - asymmetric chest expansion, dullness to percussion and diminished breath sounds - have extremely poor sensitivities and specificities for the diagnosis of pleural fluid and should not be relied on for diagnosis. ${ }^{11,12}$ In addition, it can be extremely difficult to elicit these physical examination findings in critically ill or trauma patients.

Although CXR is the most commonly used modality to detect pleural effusion, it is only able to detect relatively large effusions. Studies have shown that approximately $175 \mathrm{~mL}$ of fluid is required to cause blunting of the costophrenic angles in an erect CXR, and sometimes as much as $500 \mathrm{~mL} .{ }^{13}$ Supine CXR, used in critically ill and trauma patients, is even poorer at detecting pleural effusions: able to detect $175-525 \mathrm{~mL}$ of pleural fluid. ${ }^{14}$ The sensitivity and specificity of CXR for pleural fluid diagnosis is relatively poor. In ICU patients with coexisting lung pathology (which represents the majority of critically ill patients), 


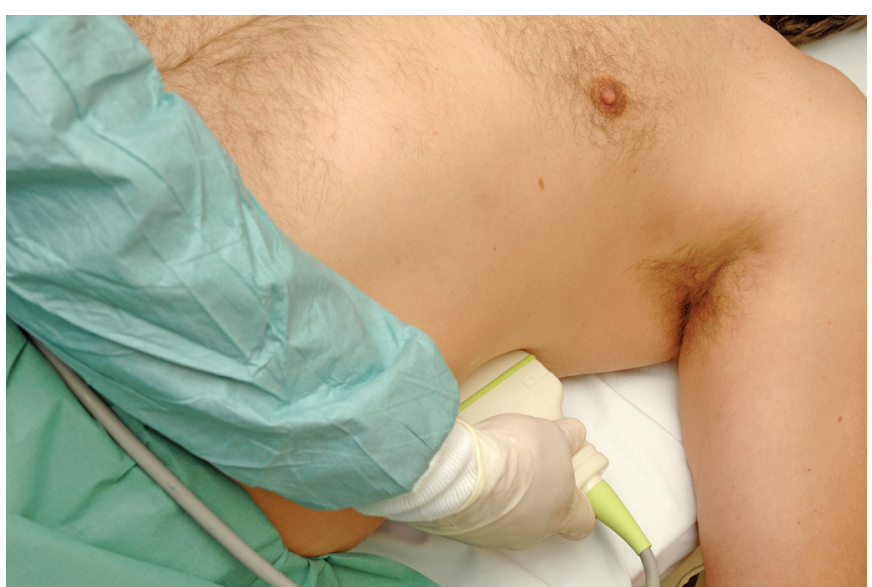

Figure 1 Probe position for pleural fluid detection in the supine patient

supine CXRs have a sensitivity of $39 \%$ and specificity of $85 \%$ for detection of pleural fluid. ${ }^{14}$ Placing patients in the lateral decubitus position improves detection of pleural fluid. ${ }^{15,16}$ However, this is often impractical in the critically ill patient. In thoracic trauma patients, the sensitivity and specificity of supine CXR is better, with sensitivities of $92-96 \%$ and specificities nearing $100 \%$. 4

The significance of pleural fluid to the patient's presentation is not always clear, and so early detection of pleural fluid is important to guide the decision to drain, either for diagnostic or therapeutic purposes.

\section{Case example \#1}

A 25-year-old man presents following a high speed motor vehicle collision, in which he was the belted driver. He is brought into the ED by ambulance, supine, with cervical spine immobilization. He is haemodynamically stable and complaining of shortness of breath. After primary and secondary surveys are performed, as outlined by advanced trauma life support protocol, ${ }^{17}$ ultrasound is used to examine the lungs, and determines the presence of a large fluid collection in the right chest. Based on this finding, a chest tube is placed; $1 \mathrm{~L}$ of blood is quickly drained, with resolution of the patient's symptoms. As the chest tube is being secured, the $X$-ray technician arrives with the portable X-ray machine to perform the X-ray that was called for on arrival of the patient.

\section{Case example \#2}

A 70-year-old woman with a long smoking history presents to the emergency department complaining of a gradual onset of shortness of breath. Investigations show only a small pleural effusion on CXR. Suspecting that this is a malignant effusion, ultrasound-guided thoracentesis is performed. A sample is obtained without complication and sent for analysis.

\section{Evidence}

The use of ultrasound for the detection of pleural fluid was first described in $1967 .{ }^{18}$ Since that time, ultrasound has been shown to be extremely sensitive for the detection of pleural fluid, with the ability to detect as little as $20 \mathrm{~mL}$. $7,19,20$

The clinical sensitivity and specificity of PoCUS for the detection of pleural fluid has also been demonstrated. In the thoracic trauma population, PoCUS has consistently been shown to have a sensitivity between $92 \%$ and $100 \%$ and a specificity nearing $100 \%$ for haemothorax. ${ }^{4-10}$ In the ICU population, PoCUS has demonstrated a sensitivity of $92 \%$ and specificity of $93 \%$ for the detection of pleural fluid, ${ }^{14}$ even in the presence of severe pulmonary pathology.

In addition to an excellent sensitivity and specificity for detection of pleural fluid, PoCUS drastically reduces the time to clinical diagnosis when compared with CXR. In the trauma population, PoCUS has been shown to provide a diagnosis of haemothorax within one minute compared with 15 minutes by CXR. ${ }^{4,6}$ The clinical impact of the use of PoCUS for the detection of pleural fluid is unclear, however, as it has not been studied in any clinical trials to date.

PoCUS can also aid thoracentesis. Use of PoCUS has been shown to increase the success of thoracentesis, while simultaneously decreasing the complication rate..$^{21-23}$ In a small randomized control trial with 52 patients, the failure rate in clinically-guided thoracentesis was 33\% compared with $0 \%$ with ultrasound guidance. ${ }^{21}$ Two large retrospective cohort studies with 342 and 523 patients showed that ultrasound guidance reduced the rate of pneumothorax from thoracentesis by more than half (from $18 \%$ to $3 \%$, and from $10.3 \%$ to $4.9 \%$ respectively). ${ }^{22,23}$ The benefits of ultrasound guidance for thoracentesis have led the British Thoracic Society to recommend the routine use of ultrasound guidance for thoracentesis. ${ }^{24}$

\section{Image generation - how to get the right image}

\section{Probe selection and machine settings}

When image interpretation requires deep image penetration, a phased array (cardiac) transducer or a low frequency $(3-5 \mathrm{MHz})$ curvilinear (abdominal) transducer is preferable. For superficial effusions, a linear (vascular) transducer with higher frequency $(5-10 \mathrm{MHz})$ is used. The machine can be set to abdominal pre-sets.

\section{Patient position, surface anatomy and key landmarks}

The examination may be done with the patient in the supine or erect positions, as determined by the clinical scenario. The transducer should be placed in the most dependent area, as that is where fluid will collect - inferior chest if the patient is in an erect position, and posterolaterally in the supine patient (Figure 1). Optimizing patient position enhances procedural success. Elective thoracentesis is often performed with the patient in the erect position, leaning forward, with arms crossed in front. This allows the effusion to collect at the lung bases. The ultrasound probe can then be placed on the back in the posterior axillary line to image the best location for drainage. Critically ill patients 


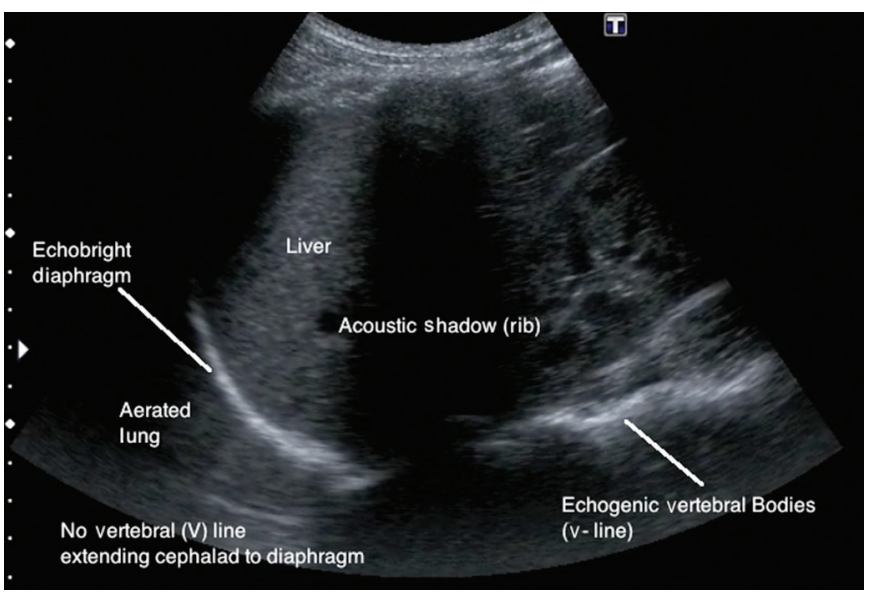

Figure 2 Identification of the diaphragm in relation to the liver. The diaphragm is found immediately superior to the liver on the right side and the spleen on the left side

are often required to remain in the supine position. A moderate effusion can still be well visualized by scanning the lateral chest wall at the posterior axillary line. The head of the bed may need to be raised, if possible, to visualize smaller effusions. Elevating the patient's arm over the head increases the distance between ribs, helping to improve image generation. Where this is not practical the ipsilateral arm can be pulled across the chest to the opposite side.

Greatest success is achieved when the transducer is placed immediately superior to the diaphragm. ${ }^{25}$ The diaphragm is generally found at the level of the xiphoid process, and is perhaps most easily identified by its close relationship with the spleen and liver. The diaphragm is immediately superior to the spleen or liver and can easily be found using these organs as landmarks (Figure 2).

While effusions can be found at any level of the pleural cavity, the diaphragm is the best starting point to search for pleural fluid. One can examine more superior aspects of the pleural cavity for fluid using the pleural line as the landmark. When looking for pleural fluid, the most dependent areas of the chest are scanned as this is where fluid will collect, in contrast to pneumothorax, where the opposite is the case.

\section{Image interpretation - is there fluid in the pleural cavity?}

In the normal individual, air in the lung parenchyma scatters the ultrasound beam, creating a very indistinct image (Figure 2). The diaphragm appears brightly echogenic on expiration, as it lies directly adjacent to air filled lung parenchyma, causing scattered reflection, and will disappear during inspiration as aerated lung moves to lie between it and the probe. This loss of image during inspiration is known as the lung curtain (see Supplementary Video 1).

When pleural fluid is present, an anechoic (dark) area is seen superior to the diaphragm (Figure 3 and Supplementary Video 2). In areas where the diaphragm is not present, the fluid is seen between the pleural line and lung parenchyma (Figure 4, described in more detail with discussion of the quad sign later in the text).

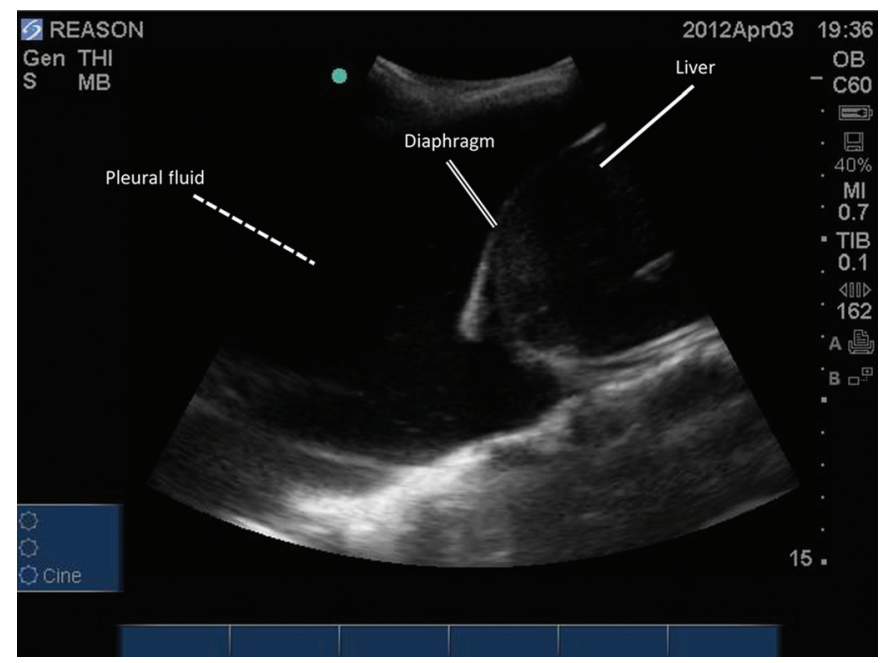

Figure 3 Pleural effusion seen as anechoic fluid (dashed line) superior to diaphragm. Note the location of the liver (solid white line), which is an easy landmark for the identification of the diaphragm (double line)

Pleural fluid greatly improves visualization of the diaphragm, which can be almost entirely seen in larger effusions. The diaphragm will however appear less echogenic, when anechoic pleural fluid is present. As pleural fluid will transmit echoes to the posterior wall of the thorax, these structures, including the vertebrae, will be visualized cephalad to the diaphragm. This is known as the vertebral (V) line, and helps distinguish pleural fluid from the loss of image, or dark lung curtain, seen with normal aerated lung (see Figure 5). ${ }^{25}$

Pleural fluid, especially when exudative, may be echoic, and can be mistaken for lung parenchyma. Therefore, two other signs have been described to help detect pleural fluid more accurately - the quad sign and the sinusoid sign. ${ }^{25}$

The quad sign refers to the visualization of an effusion between four regular borders - the pleural line, two rib shadows and the lung line (Figure 4$){ }^{26}$

There are occasions where pleural thickening may appear hypoechoic and resemble a small pleural effusion. The sinusoidal sign may be useful to distinguish between this and fluid. The sinusoid sign is seen in M-mode and demonstrates the movement of lung parenchyma in and out of the effusion during respiration - towards the periphery in inspiration and vice versa in expiration. This gives the appearance of a sinusoidal wave as the lung parenchyma moves closer and farther away from the pleural line. ${ }^{26}$

\section{Factors that hinder interpretation}

Interpretation of PoCUS for pleural fluid can be hindered in the presence of alveolar consolidation, or rib shadows, which can give the appearance of fluid. ${ }^{27}$

\section{Thoracentesis}

PoCUS can guide thoracentesis once pleural fluid is found. Once pleural fluid is identified, the largest pocket of fluid should be found, and its depth noted (the British Thoracic Society recommends thoracentesis occur only when fluid 


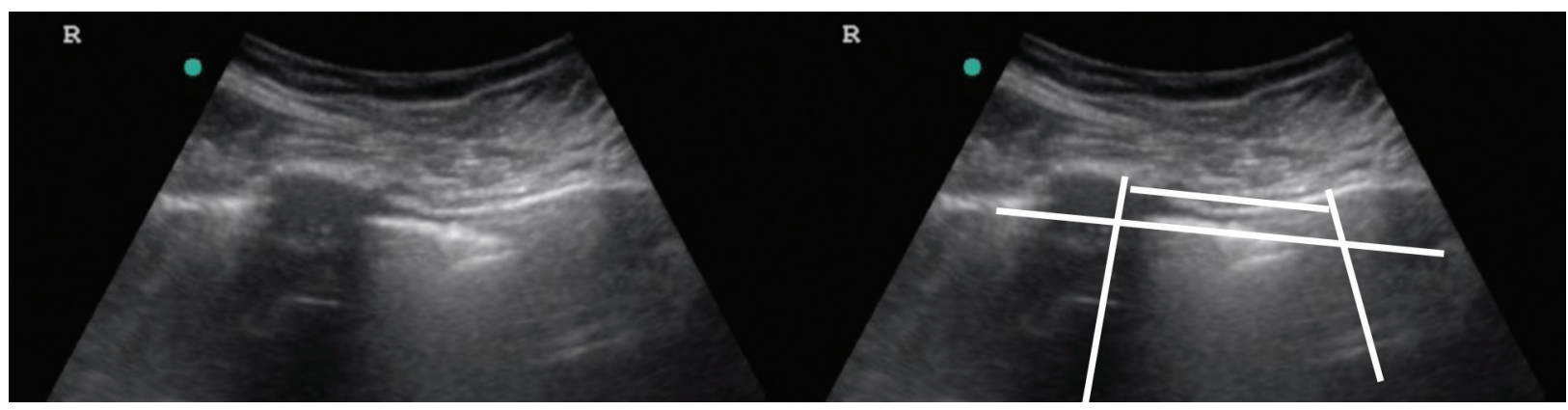

Figure 4 The quad sign. An anechoic pocket of fluid is seen contained between four borders - two rib shadows (vertical lines), the pleural line (short horizontal line) and the lung line (long horizontal line). Note that the fluid is seen deep to the plural line and superficial to the lung

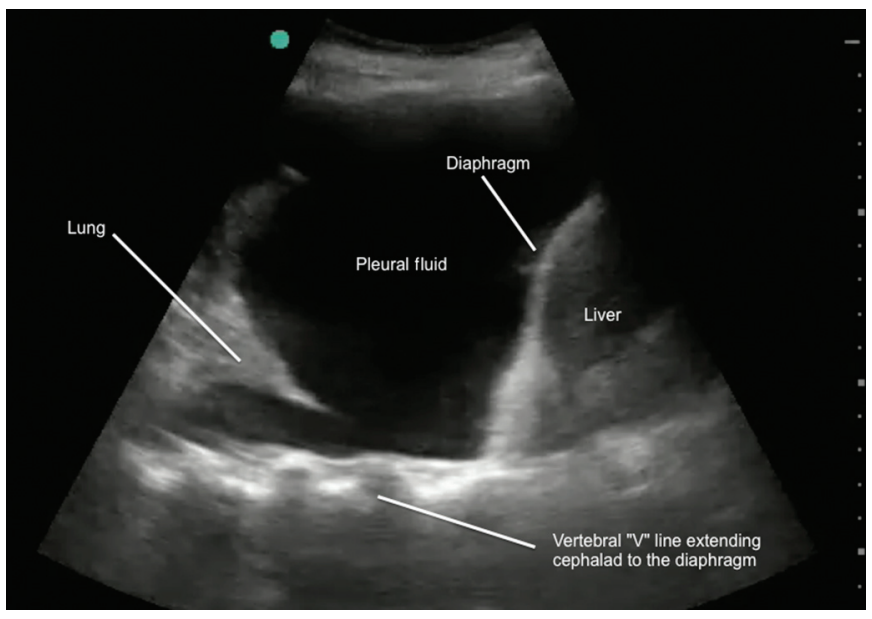

Figure 5 Visualization of the vertebral shadow above (cephalad) to the diaphragm, known as the vertebral $(\mathrm{V})$ line, is indicative of pleural fluid

pockets greater than $10 \mathrm{~mm}$ are seen). ${ }^{24}$ An ultrasoundguided thoracentesis may then proceed using a static or dynamic technique.

In the static technique, the effusion is identified using a curvilinear or phased array probe, a mark is placed on the skin over the location of the deepest area of fluid, away from the diaphragm and organs, with thoracentesis then proceeding in the usual way. The depth of the fluid is also noted and guides the depth of needle insertion. If the static technique is used, care should be taken to conduct the thoracentesis in the same position as the PoCUS was obtained, as movement of the patient will shift the fluid. There is evidence showing that the static technique may not decrease complication risk significantly compared with a blind approach when the scan is not performed at the bedside, ${ }^{28}$ likely due to fluid shifts between the time of marking and aspiration.

The dynamic technique uses realtime ultrasound guidance. Once the patient is prepared for thoracentesis in the usual way, and the deepest area of fluid is marked as described above, a linear ultrasound probe is selected, covered with a sterile cover and the needle is then inserted under ultrasound visualization, either in or out of plane, and aspiration occurs in the usual way. For the in-plane approach (Figure 6a), the needle enters the skin at the side of the probe and traverses the ultrasound beam. This technique provides a larger needle artefact, which is easier to (a)

\section{In-plane technique}

(b)
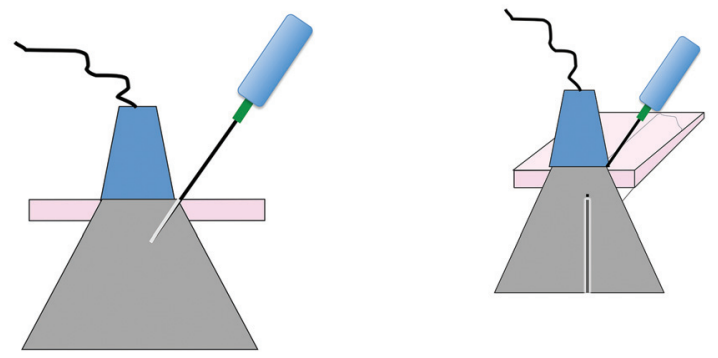

Figure 6 (a, b) The in-plane and out-of-plane techniques of ultrasound needle guidance

track. In areas where there are many confined structures, such as in the neck, it may be more difficult to see structures behind the needle. This is not a problem when performing thoracentesis. For the out-of-plane approach (Figure 6b) the needle enters the skin away from the probe and is aimed at the ultrasound beam. The needle tip intersects the ultrasound beam, which is already located over the optimal side for drainage. This is a technically more difficult manoeuver due to the reduced visual of the small needle tip.

\section{Conclusion}

PoCUS is a fast and sensitive modality for the detection of pleural fluid. The literature shows that compared with CXR, PoCUS can provide a diagnosis of pleural effusion faster, and with a superior sensitivity and specificity.

Once pleural effusion is found, PoCUS can also help to ensure the successful and safe performance of thoracentesis. The complication rate of thoracentesis performed under ultrasound guidance is less than half of that using the blind technique.

\section{DECLARATIONS}

Competing interests: None.

Funding: This research received no specific grant from any funding agency in the public, commercial, or not-for-profit sectors. 
Ethical approval: N/A.

Guarantor: Dr Atkinson.

Contributorship: OL reviewed the literature and composed the text. PA proposed the content and context, reviewed the literature and revised the text and figures. JB reviewed the literature and reviewed the text. RJ conceived the title, reviewed the literature and reviewed the text. AS reviewed the literature and reviewed the text. JT reviewed the literature and reviewed the text.

Acknowledgements: None.

\section{REFERENCES}

1 Noppen M, De Waele M, Li R, et al. Volume and cellular content of normal pleural fluid in humans examined by pleural lavage. Am J Respir Crit Care Med 2000;162(Pt 1):1023-6

2 Knudsen CW, Omland T, Clopton P, et al. Diagnostic value of B-Type natriuretic peptide and chest radiographic findings in patients with acute dyspnea. Am J Med 2004;116:363-8

3 Mattison LE, Coppage L, Alderman DF, Herlong JO, Sahn SA. Pleural effusions in the medical ICU: prevalence, causes and clinical implications. Chest 1997;111:1018-23

4 Sisley AC, Rozycki GS, Ballard RB, Namias N, Salomone JP, Feliciano DV. Rapid detection of traumatic effusion using surgeon-performed ultrasonography. J Trauma 1998;44:291-7

$5 \mathrm{Ma}$ OJ, Mateer JR. Trauma ultrasound examination versus chest radiography in the detection of hemothorax. Ann Emerg Med 1997;29:312-6

6 Ma OJ, Mateer JR, Ogata M, Kefer MP, Wittmann D, Aprahamian C. Prospective analysis of a rapid trauma ultrasound examination performed by emergency physicians. J Trauma 1995;38:879-85

7 Rothlin MA, Näf R, Amgwerd M, Candinas D, Frick T, Trentz O. Ultrasound in blunt abdominal and thoracic trauma. J Trauma 1993;34:488-95

8 Rozycki GS, Ochsner MG, Schmidt JA, et al. A prospective study of surgeon-performed ultrasound as the primary adjuvant modality for injured patient assessment. J Trauma 1995;39:492-500

9 Brooks A, Davies B, Smethhurst M, et al. Emergency ultrasound in the acute assessment of haemothorax. Emerg Med J 2004;21:44-6

10 Kimura A, Otsuka T. Emergency center ultrasonography in the evaluation of hemoperitoneum: a prospective study. J Trauma 1991;31:20-3

11 Wong CL, Holroyd-Leduc J, Straus SE. Does this patient have a pleural effusion? JAMA 2009;301:309-17

12 Lichtenstein D, Goldstein I, Mourgeon E, Cluzel P, Grenier P, Rouby JJ. Comparative diagnostic performances of auscultation, chest radiography and lung ultrasonography in acute respiratory distress syndrome. Anesthesiology 2004;100:9-15

13 Colins JD, Burwell D, Furmanski S, et al. Minimal detectable pleural effusions. A roentgen pathology model. Radiology 1972;105:51-3

14 Woodring JH. Recognition of pleural effusion on supine radiographs: how much fluid is required? AJR Am J Roentgenol 1984;142:59-64
15 Ruskin JA, Gurney JW, Thorsen MK, Goodman LR. Detection of pleural effusions on supine chest radiographs. AJR Am J Roentgenol 1987;148:681-3

16 Moskowitz H, Platt RT, Schachar R, Mellins H. Roentgen visualization of minute pleural effusion. An experimental study to determine the minimum amount of pleural fluid visible on a radiograph. Radiology 1973;109:33-5

17 American College of Surgeons Trauma Committee. Advanced Trauma Life Support for Doctors. 8th edn. Chicago, IL: American College of Surgeons, 2008

18 Joyner CR, Herman RJ, Reid JM. Reflected ultrasound in the detection and localization of pleural effusion. JAMA 1967;200:399-402

19 Walz M, Muhr G. Sonographische Diagnostik beim stumpfen Thoraxtrauma. Unfallchirurg 1990;93:359

20 Borner N, Kelbel C, Lorenz J, et al. Sonographische Volumenbestimmung und Drainage von Pleuraergussen. Ulraschall Klin Prax 1987;2:148

21 Grogan DR, Irwin RS, Channick R, et al. Complications associated with thoracentesis. A prospective, randomized study comparing three different methods. Arch Intern Med 1990;150:873e7

22 Raptopoulos V, Davis LM, Lee G, et al. Factors affecting the development of pneumothorax associated with thoracentesis. AJR Am J Roentgenol 1991;156:917e20

23 Barnes TW, Morgenthaler TI, Olson EJ, et al. Sonographically guided thoracentesis and rate of pneumothorax. J Clin Ultrasound 2005;33:442e6

24 Havelock T, Teoh R, Laws D, et al. Pleural procedures and thoracic ultrasound: British Thoracic Society Pleural Disease Guideline 2010. Thorax 2010;65(Suppl. 2):ii61-76

25 Atkinson P, Milne J, Loubani O, Verheul G. The V-line: a sonographic aid for the confirmation of pleural fluid. Critical Ultrasound J. 2012;4:19

26 Lichtenstein DA. Ultrasound examination of the lungs in the intensive care unit. Pediatr Crit Care Med 2009;10:693-8

27 Lichtenstein D. Pleural effusion and introduction to the lung ultrasound technique. In: Lichtenstein D, Pinsky M, Jardin F, eds. General Ultrasound in the Critically Ill. Berlin, Germany: Springer-Verlag, 2007:96-104

28 Kohan JM, Poe RH, Israel RH, et al. Value of chest ultrasonography versus decubitus roentgenography for thoracentesis. Am Rev Respir Dis 1986;133:1124e6

\section{Appendix}

Supplementary Video 1 Ultrasound image of normal diaphragm and lung during respiration http:/ / dl.dropbox.com/u/21386908/Webvideos/Video \% 20clip \%201\%20normal\%20lung.mov

Supplementary Video 2 Pleural effusion seen as anechoic fluid (dashed line) superior to diaphragm. Note the location of the liver (solid white line), which is an easy landmark for the identification of the diaphragm

http:/ / dl.dropbox.com/u/21386908/Webvideos/Video \% 20clip\%202.\%20pleural\%20effusion.mp4 